\title{
Cephamycins: Treatment Option for Recurrent Clostridioides difficile Infection
}

\author{
Anshul Sood
}

\author{
Journal of Gastrointestinal Infections (2019): 10.5005/jp-journals-10068-3029
}

Bacteria are known to withstand adverse conditions by differentiating vegetative cells into spores as a means of survival. Spore-forming bacteria are ubiquitous in nature ranging from natural food contaminants (Bacillus cereus), bioterrorism agents (B. anthracis) to specific representatives of Clostridium sp. as animal pathogens. Clostridium difficile, a toxin-producing spore bearer now known as Clostridioides difficile is one of the human pathogens found to be associated with antibiotic-associated diarrhea and pseudomembranous colitis. ${ }^{1}$ Literature suggests that around $35 \%$ of patients experience $C$. difficile infection relapse once the treatment with first-line of antibiotics (metronidazole and vancomycin) is stopped and even multiple relapses were observed in $40-60 \%$ of these patients. ${ }^{2,3}$ The major cause of initiation, dissemination, and reinfection of $C$. difficile disease is the formation of spores since metronidazole and vancomycin merely act upon the vegetative cells and do not possess sporicidal activity. ${ }^{3}$ Other than these, the Food and Drug Administration-approved fidaxomicin is also currently in use, but its affordability is already an ongoing debate. ${ }^{4}$ So in the present scenario when there is no $C$. difficile vaccine, it calls for an urgent need to devise economical therapeutic strategies that can control the cycle of $C$. difficile reinfection by acting not only on the vegetative cells but also on the spores.

In a recent study published in Nature Microbiology, August 2019, Srikhanta et al. ${ }^{3}$ from Monash University tested the sporicidal efficacy of cephamycins (cefoxitin, cefotetan, and cefmetazole) in bacterial pathogens. In a mouse disease model, authors used transmission electron microscopy imaging to visualize the effect of subinhibitory concentration of cephamycins on C. difficile sporulation at different days and compared them with the untreated control cultures. In cephamycin-treated cells, the absence of spores was evident, which suggested that cephamycins affect $C$. difficile sporulation negatively. A significant reduction in spores was seen in other representatives of the $C$. difficile ribotype 027 (RT027) clade 2 epidemic lineage, together with R20291 (UK), M7404 (Canada), DLL3109 (Australia), and the historical, nonepidemic RT027 strain CD196. Similar results were noticed in human isolates of other $C$. difficile clades. Also the antisporulating properties of the cephamycins were evident in other spore-forming pathogens such as Bacillus subtilis and $B$. cereus validating its broad-spectrum activity. But no significant reduction was seen in the number of vegetative cells at any time point. Among the three tested cephamycins, cefotetan represented with the strongest antisporulating activity. Antisporulating capability of cephamycins were then compared with three different cephalosporins (cefaclor, cefuroxime, and cefotaxime), and it was found that cephalosporins showed $50-80$-fold reduction in spores as compared to $10^{3}-10^{4}$ fold reduction in the presence of cephamycins. Authors have also tested the antisporulating activity of first-line antibiotic such as vancomycin which was found to have no effect on sporulation. ${ }^{3}$
Department of Medical Microbiology, Postgraduate Institute of Medical Education and Research, Chandigarh, India

Corresponding Author: Anshul Sood, Department of Medical Microbiology, Postgraduate Institute of Medical Education and Research, Chandigarh, India, Phone: +91 8146520659, e-mail: anshulsood02@gmail.com

How to cite this article: Sood A. Cephamycins: Treatment Option for Recurrent Clostridioides difficile Infection. J Gastrointest Infect 2019;9(1):57-58.

Source of support: Nil

Conflict of interest: None

Subsequently to identify the target molecules of cephamycins, the team used fluorescently labeled penicillin-derivative bocillin-FL (Boc-FL) as a competitive inhibitor of cephamycin. Membrane proteins of cephamycin-treated and untreated cells were isolated and later exposed to Boc-FL. Since Boc-FL is a competitive inhibitor of cephamycin, it competed for the same $\beta$-lactam-binding site to which cephamycin was bound. Hence when visualized, membrane proteins of cephamycin-treated cells showed reduced Boc-FLbinding sites and three proteins were exclusively found absent in treated cells but were present in untreated cells. These three proteins (CdspoVD, Cdpgt, and CddacF) were identified as a subset of penicillin-binding proteins by mass spectrometry that had earlier been implicated in C. difficile sporulation. To phenotypically confirm their importance in sporulation, authors carried out gene transfer experiments by TargeTron mutagenesis. It was observed that the CdspoVD gene knockout completely affected the spore-forming capability of CdspoVD mutant and impaired the expression of other two genes (Cdpgt and CddacF) as well. In Cdpgt mutant, the antisporulating activity was not completely impaired but sporulation was reduced between 21- and 123-fold, whereas CddacF mutation had no effect on sporulation. This clearly stated that CdSpoVD was the prime antisporulating target of the cephamycins, which in future could be exploited as a target for drug development. ${ }^{3}$

Till now it was seen that vancomycin is the primary standard of care and does not have any effect on sporulation, whereas cephamycins do not act against vegetative cells. Therefore, in another set of experiment, authors coadministered vancomycin with different concentrations of cephamycin (cefotetan) to check the activity against Clostridioides difficle infection (CDI) relapse and it was noticed that cotreatment with vancomycin and cefotetan $(50 \mu \mathrm{g} / \mathrm{mL})$ prevented CDI relapse remarkably. Therefore, the combination of drugs demonstrated in this study appears to be a promising therapeutic for $C D I$ relapse and while $C$. difficile vegetative cells show resistance to cephamycins, other spore-bearing pathogens may have different set of penicillin-binding proteins sensitive to cephamycins where the drug alone can be used as an effective antisporulating agent.

(c) The Author(s). 2019 Open Access This article is distributed under the terms of the Creative Commons Attribution 4.0 International License (https://creativecommons. org/licenses/by-nc/4.0/), which permits unrestricted use, distribution, and non-commercial reproduction in any medium, provided you give appropriate credit to the original author(s) and the source, provide a link to the Creative Commons license, and indicate if changes were made. The Creative Commons Public Domain Dedication waiver (http://creativecommons.org/publicdomain/zero/1.0/) applies to the data made available in this article, unless otherwise stated. 


\section{References}

1. Bartlett JG, Moon N, Chang TW, et al. Role of Clostridium difficile in antibiotic-associated pseudomembranous colitis. Gastroenterology 1978;75(5):778-782. DOI: 10.1016/0016-5085(78)90457-2.

2. Hopkins RJ, Wilson RB. Treatment of recurrent Clostridium difficile colitis: a narrative review. Gastroenterol Rep 2018;6(1):21-28. DOI: 10.1093/gastro/gox041.
3. Srikhanta YN, Hutton ML, Awad MM, et al. Cephamycins inhibit pathogen sporulation and effectively treat recurrent Clostridioides difficile infection. Nat Microbiol 2019;4(12):2237-2245. DOI: 10.1038/ s41564-019-0519-1.

4. Lam SW, Neuner EA, Fraser TG, et al. Cost-effectiveness of three different strategies for the treatment of first recurrent Clostridium difficile infection diagnosed in a community setting. Infect Control Hosp Epidemiol 2018;39(8):924-930. DOI: 10.1017/ice.2018.139. 